\title{
Assessment of Traffic Impact on Future Cooperative Driving Systems: Challenges and Considerations
}

\author{
Fei Liu ${ }^{1}$, Rattaphol Pueboobpaphan ${ }^{1}$, and Bart van Arem ${ }^{2}$ \\ ${ }^{1}$ Applications of Integrated Driver Assistance (AIDA), Centre for Transport Studies, University of Twente, the Netherlands \\ ${ }^{2}$ Civil Engineering and Geosciences, Transport \& Planning, Delft University of Technology, the Netherlands
}

\{f.liu, r.pueboobpaphan\}@utwente.nl, b.vanarem@tudelft.nl

\begin{abstract}
Connect \& Drive is a start-up project to develop a cooperative driving system and improve the traffic performance on Dutch highways. It consists of two interactive subsystems: cooperative adaptive cruise control (CACC) and connected cruise control (CCC). To assess the traffic performance, a traffic simulation model will be established for large-scale evaluation and providing feedbacks to system designs. This paper studies the factors determining the traffic performance and discusses challenges and difficulties to establish such a traffic simulation model.
\end{abstract}

Keywords-CACC; cooperative driving; traffic simulation; traffic flow stability

\section{INTRODUCTION}

In the Netherlands, traffic density increases every year [1]. According to [2], from 2000 till 2020, passenger traffic will have an expected growth of 15 to $40 \%$, and freight traffic will grow between 15 to $80 \%$, depending on economic developments. This leads to higher traffic density and busier roads. Traffic congestions and safety become two very important concerns of Dutch motorways. Besides improving safety policies, roadside infrastructure, and traffic education, latest technologies are also expected to contribute to solve the problems. Various components can be equipped in vehicles and road side units to improve vehicle safety and comfort, traffic safety and efficiency, such as communication systems, controllers, sensors, navigation systems. We name such systems as advanced driver assistance systems (ADA). ADA systems control and manage the speed and lane change of vehicles. Some of them also provide extra information, such as the distance to the preceding vehicle, traffic sign recognition, blind spot detection, etc., to avoid traffic collisions and accidents.

One of the important research directions within ADA is cruise control, which can automatically control the speed of a vehicle. The advanced version of cruise control is adaptive cruise control (ACC). An ACC vehicle is generally equipped with a radar or laser to detect speed information from the preceding vehicle. The vehicle adjusts its speed accordingly based on the detection information.

Based on ACC, a further improved version, cooperative adaptive cruise control (CACC), is proposed. In such a system, vehicles can not only sense the information from the preceding vehicle, but also communicate with other vehicles (V2V) and infrastructure (V2I) within communication range. After coordinating all information, vehicles make decisions of acceleration, deceleration, or keeping the current speed.

Other than fully automatic controlled system CACC, there is another advisory advanced cruise control system called connected cruise control (CCC). In CCC systems, vehicles are also equipped with wireless communication facilities and have information processing and coordination abilities. However, vehicles do not have the responsibilities of taking actions, while drivers do. Advices of cooperative driving are given to the drivers via human-machine interface (HMI). Drivers have the freedom to take or not the required cooperative action.

Connect \& Drive (C\&D) is an ADA system research project starting from January 2009. It is funded by High Tech Automotive System (HTAS) in the Netherlands. This project aims to design and develop new generation vehicles equipped with ADA systems in order to improve the current traffic congestions, the road capacity, and safety in the Netherlands. Study shows that conventional ACC systems have limited impacts on the traffic flow $[3,4]$. We believe with more and better information gathered through wireless communication from other vehicles and RSUs, more precise advices or actions can be taken to smooth the traffic jams. Drivers can gain longer anticipation time. Vehicles can better coordinate and cooperate with each other. Therefore, C\&D project focuses on CACC and $\mathrm{CCC}$ systems to improve the road condition. Three basic traffic situations of platoons attract our special interests [5]:

- Increase the size of a platoon, especially when vehicles cut into a platoon, for example, at merging junctions, lane drop, and etc.

- Decrease the size of a platoon, especially when vehicles cut out from a platoon, for example, at existing splits, lane widening, and etc.

- Sudden changes in a platoon, especially when one or more vehicles brake, accelerate, decelerate, and incidents.

A prototype of vehicles equipped with $\mathrm{CACC}$ and $\mathrm{CCC}$ systems will be set up for real field testing. However, real-field vehicles test can only be implemented small-scale due to the cost and safety reasons. The test is not sufficient to demonstrate all the possible situations in highways. Further, in some cases, it is not enough to observe the impacts, such as shockwave mitigation, on the entire traffic with small-scale tests. Therefore, traffic simulation will be used to analyze the traffic performance from macroscopic perspective. 
This paper aims to discuss the traffic simulations for $\mathrm{C}(\mathrm{A}) \mathrm{CC}$ system. We will reveal the factors which influence the traffic performance and discuss the challenges and difficulties to establish a traffic simulation model for $\mathrm{C}(\mathrm{A}) \mathrm{CC}$ systems.

This paper is organized as follows. In Section 2, we introduce some related CACC projects and present our research interest. In Section 3, we discuss the relation between driver/vehicle characteristics and platoon/traffic flow stability. In Section 4, we focus on the challenges and difficulties of the simulation in CACC/CCC-based traffic systems. In Section 5, we summarize the paper and present our future work.

\section{PROBLEM DESCRIPTIONS AND RESEARCH OBJECTIVES}

The existing literatures of CACC proposed various designs of CACC systems. Some traffic simulation models have been established under different circumstances. For instance, Van Arem, et al. modeled a 4-lane highway with a bottleneck of lane drop with the traffic simulator MIXIC [17]. A CACC system with communication between vehicles is proposed. The results show the potential positive impact of CACC on traffic throughput and stability, especially near a lane drop. $\mathrm{Xu}$ simulated the ACC/CACC vehicles merging in highways [18] with V2V and V2I communications. The studies show that the higher market penetration of ACC/CACC vehicles, the better traffic performance in terms of velocity and braking effort. CACC also shows better results than ACC in all tests. Similar topic has also been studied in [19]. A model of a single highway lane with junctions has been simulated. Different market penetration of $\mathrm{ACC}$ and $\mathrm{CACC}$ vehicles has been examined. The results show that CACC can potentially double the capacity of a highway lane with a high CACC market penetration.

The reviewed literatures show that CACC systems have positive impact on the traffic throughput. However, CACC algorithms are still under development. There exists no standard design yet. In general, a vehicle can gain a better overview of the traffic environment around, if more information about traffic can be obtained from other vehicles or infrastructures. The better overview of surrounding traffic situation, the better decision a vehicle can make. Moreover, the current simulation models do not take into account communication factors, such as delay, packet loss, signal fading, etc. Further, current studies focus on longitudinal control and highway merge.

In the $\mathrm{C} \& \mathrm{D}$ project, $\mathrm{C}(\mathrm{A}) \mathrm{CC}$ algorithms will be developed to cope with situations like forming platoons, sudden changes, cut-in, cut-out, merge, and exit. Special communication protocols between vehicles and infrastructures are designed to facilitate the system. We need to establish a traffic simulation model especially for the new proposed $\mathrm{C}(\mathrm{A}) \mathrm{CC}$ systems with the abstract communication model to assess the traffic performance under mentioned scenarios.

There are three major motivations to establish traffic simulations on proposed $\mathrm{C}(\mathrm{A}) \mathrm{CC}$ algorithms in $\mathrm{C} \& \mathrm{D}$ project. First of all, as we mentioned before, traffic simulations can help us to study the impact of our $\mathrm{C}(\mathrm{A}) \mathrm{CC}$ algorithms at traffic flow level, to compensate the weakness of small-scale real field test. On the other hand, traffic simulations can support system designs by giving feedbacks of simulation results. The simulation results can be used as a guideline of adjusting the current system design. Finally, the simulation model will be used to evaluate the real field test results and the final design of our C(A)CC system.

The traffic simulation defines the individual behaviors of each vehicle based on proposed $\mathrm{C}(\mathrm{A}) \mathrm{CC}$ algorithms and analyzes the traffic flow performance with large-scale scenarios. It needs to consider the following research objectives:

- To establish a $\mathrm{C}(\mathrm{A}) \mathrm{CC}$ traffic simulation model with an abstract analytical $\mathrm{V} 2 \mathrm{~V}$ and V2I communication model and an analytical human-in-the-loop control model.

- To study the impact of $\mathrm{C}(\mathrm{A}) \mathrm{CC}$ on traffic flow performance.

- To provide design requirements for $\mathrm{C}(\mathrm{A}) \mathrm{CC}$ based on traffic flow consideration.

- $\quad$ To generate detailed testing scenarios based on $\mathrm{C} \& \mathrm{D}$ project scopes.

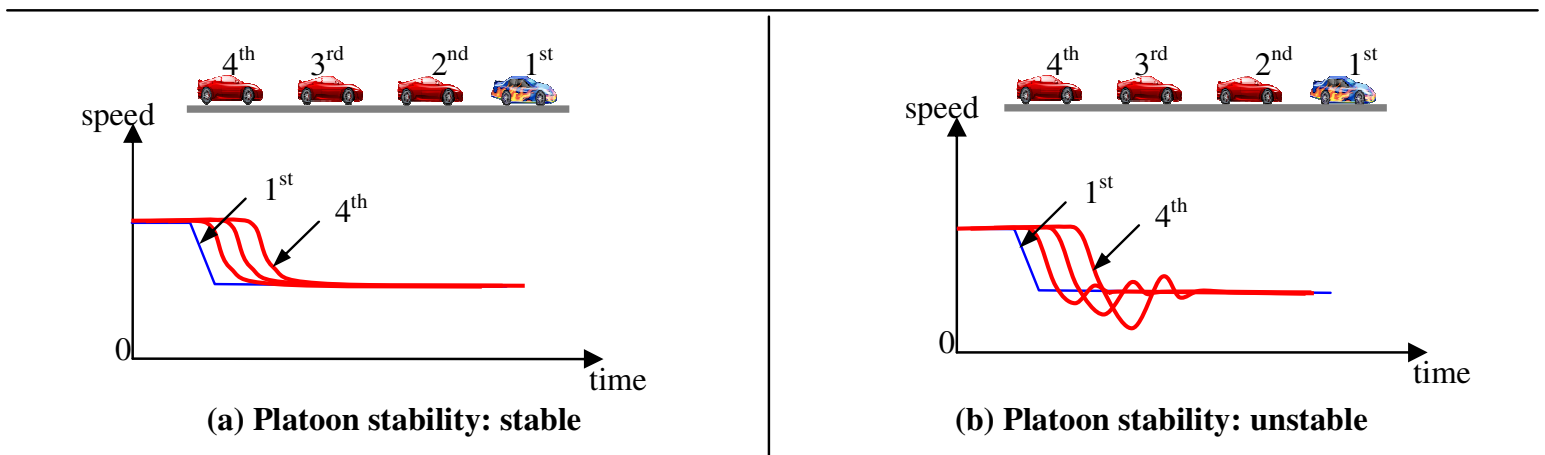

Figure 1. Stability concepts. A platoon of vehicles follows the blue leader in (a) and (b). The platoon in (a) is considered as platoon stable while in (b) as platoon unstable. 


\section{DRIVER/VEHICLE CHARACTERISTICS AND PLATOON/TRAFFIC FLOW STABILITY}

Before we establish a traffic simulation model to evaluate traffic flow performance, it is first necessary to investigate what are the factors that influence the traffic flow performance and what are the relationships in between. In this section, we will investigate those essential factors and their relations to the behaviors of platoon and traffic flow.

In general, a well performed traffic flow should first be smooth and stable. A stable dynamical system is the one that, when perturbed from an equilibrium state, tends to return to that equilibrium state. In traffic engineering, the term platoon stability is concerned with a propagation of the disturbance from one vehicle to other vehicles in the same platoon. If the magnitude of the disturbance grows as it propagates to the vehicles in the upstream, the platoon is said to be unstable. When consider at the traffic stream level (which may consist of several platoons), the term traffic flow stability is used. Traffic flow stability can be determined by the stability of the platoons as well as the distribution of platoon sizes and the distribution of the inter-platoon gaps [6]. Example illustration of stable and unstable platoon is shown in Fig. 1(a) and 1(b), respectively.

Traffic flow stability has a crucial role on the overall performance of the traffic stream. Empirical results have shown that manual traffic is generally unstable [7-9] and could lead to spontaneous congestion, even in an absence of a bottleneck [10]. A recent experiment from Japan on a circular track has confirmed this phenomenon and emphasized the role of instability in traffic congestion [11]. It appears that individual driving behavior and vehicle characteristics are key determinant factors that determine the stability of the platoon and traffic flow $[6,12]$. The relation could be summarized as shown in Fig. 2.

From Fig. 2, platoon stability is dependent on driver behavior, vehicle characteristics, and the disturbance. Driver/vehicle characteristics that could affect the stability are, for example, driver reaction time, driver anticipation, desired spacing or time headway, vehicle relaxation time, acceleration capability. The amplitude of the disturbance was also found to have an effect on the stability of the platoon. Traffic flow stability could be viewed as a more generalized one that takes both platoon stability and traffic stream characteristics into consideration. In this case traffic stream characteristics refer to the distribution of the platoon length, the inter-platoon gap, and the exact order of (de)stabilizing vehicles. After all, traffic flow stability could translate into the performance of traffic flow as one would expect that the more stable the traffic flow, the smoother and better traffic flow performance. As a result, it is expected that if we could influence driver and vehicle characteristics in a suitable way, the stability of traffic flow and the overall traffic performance could be improved. The reader is referred to Pueboobpaphan and Van Arem [13] for more information about this subject and its implication for the design of Cooperative Adaptive Cruise Control.

\section{Challenge AND DifFICUlTiES}

C\&D will develop a complete $\mathrm{C}(\mathrm{A}) \mathrm{CC}$ controller with communications system, and even human-machine interface. To simulate such a system in a traffic simulator and evaluate it, we face quite few challenges as follows.

- To choose right simulation tool. To accomplish all the research objectives mentioned above, we need a simulation tool which can support simulations in microscopic detail. We need to model each vehicle as a unique entity with its own goals and behavior characteristics. We also need to have insights of how original traffic behaviors have been defined in the simulator to provide us enough knowledge for assessment. For instance, we have set up the following simple merging example in Paramics v5.2 [15].

Example: the map consists of two lanes: one single-lane major motorway and one single-lane minor motorway joining the major way with a 250-meter long ramp, as shown in Fig 3.

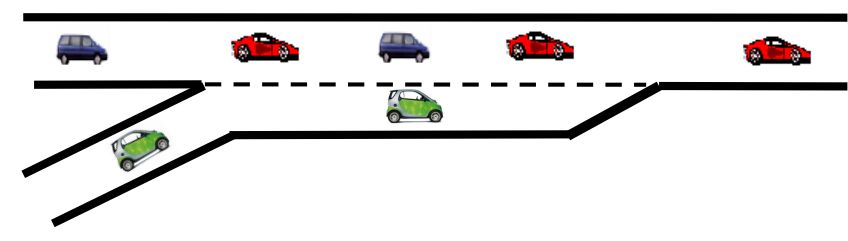

Figure 3. Merging in the highway

In Paramics v5.2, with the default settings for merging in a ramp as shown in Fig. 3, the decision for a vehicle to merge into a main lane does not only depend on the aggressiveness and time headway of vehicles, but also depends on the speed

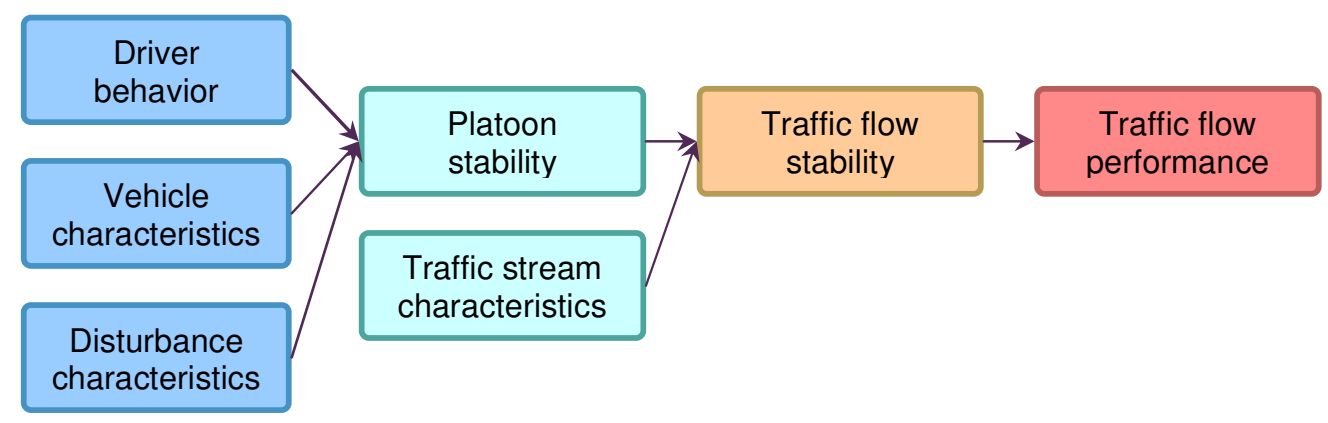

Figure 2. Relation between driver/vehicle characteristics, platoon/traffic flow stability, and traffic flow performance 
differences between the vehicles in the main lane and the merging lane. If the speed difference is too high, the vehicles in the main lanes will not slow down and create a space for the merging vehicle. Merging vehicles have to wait till the end of the main lane platoon to join the highway. The queue for merging vehicles gets longer and longer once one merging vehicle has to stop when main lane traffic is busy. Of course, this is not a realistic and desired merging reference case. The standard way to define an on-ramp merge does not work as we expected in Paramics v5.2. Different approaches have to be taken here. One simple working alternative is to code the acceleration lane as part of the main link instead of a ramp. The section of highway with the merging area will have one more lane for the merging vehicle to travel in. Further, the merging lane should be coded with restrictions of turning to make sure the merging vehicles should start merging along the entire link, instead of the end of the lane.

Because we lack of insight knowledge about the fundamental theories used to accomplish merging in Paramicsv5.2, we choose ITS modeler [14] as our simulation tool, which is developed by our cooperative institute TNO [16]. With ITS modeler, we have all freedom to define microscopic level of details. Extended functions of ITS modeler will be defined and implemented to fulfill the requirements of our $\mathrm{C}(\mathrm{A}) \mathrm{CC}$ algorithms and communication system.

- To model the communication system. One direct solution to model the communication systems into our traffic simulation is to build a platform to let two systems interact with each other real time. OMNET++ is the simulator chosen for communication simulations in this project. ITS modeler is a chosen tool for traffic simulation. There is no ready direct platform to interact between OMNET++ and ITS modeler. Those two simulators, OMNET++ and ITS modeler, are working in the different time step levels. To build such a platform to translate simulation results real-time between two simulators is very time consuming. Due to the limited time of the project, we will abstract the wireless communication system into analytical formulas and insert it to our traffic simulation model. The traffic simulation model will help to assess the communication design and provide feedbacks. However, the abstract models might not provide accurate results. We still need to investigate the accuracy of such a simulation model.

To model the human reaction factor. Human reaction factor is one parameter that we could not have direct influence on in the entire control loop. Drivers' reaction towards system suggestions might differ a lot depends on drivers' age, gender, characteristics, personal situation, weather, and so on. One of the work packages in C\&D project will study these impacts. Similar to the interface with communication systems, we will embed abstract analytical studies of human factor impact into our traffic simulation.

To build a good reasonable performed reference scenario for assessment. For the C\&D project, we are lack of existing trajectories. It is crucial to build a reference scenario with realistic demands, density, and trajectories. Within the concept of $C \& D$ project, we would like to compare our $\mathrm{C}(\mathrm{A}) \mathrm{CC}$ system with manual driving systems, which means there is no cruise control or communication ability available in vehicles.

- To define indicators for assessment of traffic performance. As we mentioned before, according to our study, traffic flow stability has a direct influence to traffic performance. A stable traffic flow can provide much better performance than an unstable one. Moreover, traffic flow stability can be evaluated with the average speed, the number of shockwaves, etc.

- To define proper test scenarios. Among the factors in determining stability of traffic flows, drivers and vehicles characteristics can be controlled some how. However, there are still some out-of-controlled key factors like market penetration rate of ADA-facilitated vehicles in a mixed traffic and the positions of ADA-facilitated vehicles in a platoon. Depending on the different settings of traffic scenarios, those factors might have both positive and negative effects on the traffic flow stability. That raises another research question: how to define proper test scenarios to evaluate our system? In this case, systematic studies of different combinations of those factors are necessary. Traffic flows are composed randomly in reality. The system behavior needs to be inspected under all different situations.

\section{CONCLUSIONS}

Connect \& Drive is going to design and implement a fully intelligent traffic system with set of interactive subsystems like cooperative adaptive cruise control (CACC) and human-in-theloop connected cruise control systems (CCC). Vehicles or the drivers of vehicles make cooperative driving decisions based on communication information from other vehicles (V2V) and infrastructures (V2I). We believe such a system can cope with many challenges and problems in nowadays traffic flow. It is expected to enhance the traffic capacity and improve the road safety and comfort.

Prototypes in a small-scale scope will be implanted at the end of the project. Although prototypes can provide us the performance of our system in the real world, we still need assessment in large-scale field. Therefore, we will build a traffic simulation model to evaluate traffic performance in macroscopic criteria, to provide feedbacks to system designers, and at the end to enhance the entire system. Based on our study, we reveal that traffic flow stability have direct influence on traffic flow performance. Moreover, it depends on various factors, such as driver/vehicle characteristics, and traffic stream characteristics. Further, we need to cope with the challenges and difficulties while building up the traffic simulation model for $\mathrm{C} \& \mathrm{D}$ project, such as traffic simulation model with communication systems and human reaction factor, build up realistic reference cases, define right performance indicators, and so on. In the coming future, we will solve the discussed problems, build the traffic simulation model, and accomplish the performance evaluation of our $\mathrm{C}(\mathrm{A}) \mathrm{CC}$ system.

\section{REFERENCES}


[1] Statistics Netherlands, available on http://www.cbs.nl/enGB/menu/home/default.htm

[2] Ministry of Transport, Public Works and Water management, Road Safety Strategic Plan 2008-2020, avaialbe on http://www.verkeerenwaterstaat.nl/english/Images/strategischplanE_tcm249-249506.pdf

[3] S. Shladover, J. VanderWerf, M.A. Miller, N. Kourjanskaia, H. Krishnan "Development and Performance Evaluation of AVCSS Deployment Sequences to Advance from Today's Driving Environment to Full Automation" August 2001). California Partners for Advanced Transit and Highways (PATH). Research Reports: Paper UCB-ITSPRR-2001-18; http://repositories.cdlib.org/its/path/reports/UCB-ITSPRR-2001-18

[4] H. Simon "Automated Highway Systems: Platoons of Vehicles Viewed as a Multiagent System" 2005, URL: www.theses.ulaval.ca/2005/22729/22729.pdf

[5] Cooperative Adaptive Cruise Control Based on Wireless Communication between Vehicles and Infrastructure WP1 report, version 15 April 2009.

[6] Tampere, C., Human-kinetic multiclass traffic flow theory and modeling: with application to advanced driver assistance systems in congestion, Doctoral dissertation, Delft University of Technology, the Netherlands, 2004.

[7] Treiterer, J., J.A. Myers, The hysteresis phenomenon in traffic flow, Proceedings of the $6^{\text {th }}$ International symposium on transportation and traffic theory, pp. 13-38, 1974.

[8] Ranjitkar, P., T. Nakatsuji, Y. Azuta, G.S. Gurusinghe, Stability analysis based on instantaneous driving behavior using car following data, Transportation Research Record: Journal of the Transportation Research Board, Vol. 1852, pp. 140-151, 2003.

[9] Ranjitkar, P., T. Nakatsuji, A. Kawamura, Experimental analysis of carfollowing dynamics and traffic stability, Transportation Research
Record: Journal of the Transportation Research Board, Vol. 1934, pp 22-32, 2005

[10] Kerner, B.S., Experimental features of self-organization in traffic flow, Physical review letters, vol. 81, no. 17, pp. 3797-3800, 1998.

[11] Sugiyama, Y., M. Fukui, M. Kikuchi, K. Hasebe, A. Nakayama, K. Nishinari, S. Tadaki, S. Yukawa, Traffic jams without bottlenecksexperimental evidence for the physic al mechanism of the formation of a jam, New journal of physics, Vol. 10, No. 3, 2008.

[12] Ossen, S. Longitudinal driving behavior: Theory and empirics, Doctoral dissertation, TRAIL Thesis series T2008/8, 2008.

[13] Pueboobpaphan, R., Van Arem, B., Understanding the relation between driver/vehicle characteristics and platoon/traffic flow stability for the design and assessment of Cooperative Adaptive Cruise Control, submitted to the 89th Transportation Research Board (TRB) annua meeting, 2010 .

[14] ITS http://www.tno.nl/downloads/ITS\%20modeller.pdf

[15] Paramics, available on http://www.paramics-online.com.

[16] TNO, http://www.tno.nl

[17] Van Arem, B., van Driel, C.J.G, Visser, R., The Impact of Co-operatie Adaptive Cruise Control on Traffic Flow Characteristics, IEEE Transactions on Intelligent Transportation Systems, Vol. 7, p.429-436, 2006.

[18] Xu, Q., Sengupta, R., Simulation, Analysis, and Coparison of ACC and CACC in Highway Merging Control, Proceedings of Intelligent Vehicles Symposium 2003, pp. 237-242, June 2003

[19] Vander Werf, J., Shaldover, S.E., Miller, M.A., Kourjanskaia, N., Evaluation of the Effects of Adaptive Cruise Control Systems on Highway Traffic Flow Capacity and Implications for Deployment of Future Automated Systems", Transportation Research Record 1900, pp. 78-84, 2003. 